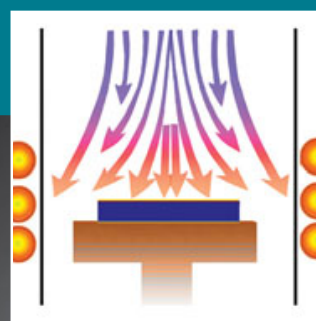

\title{
Two-dimensional layered transition-metal dichalcogenides for versatile properties and applications
}

\author{
Eric M. Vogel and Joshua A. Robinson, Guest Editors
}

\begin{abstract}
Transition-metal dichalcogenides (TMDCs) are compounds consisting of a transition-metal $\mathrm{M}(\mathrm{Ti}, \mathrm{Hf}, \mathrm{Zr}, \mathrm{V}, \mathrm{Nb}, \mathrm{Ta}, \mathrm{Mo}, \mathrm{W}, \mathrm{Tc}, \mathrm{Re}, \mathrm{Pd}, \mathrm{Pt}$ ) and chalcogen atoms X (S, Se, Te). There are approximately 60 compounds in the metal chalcogenide family, and two-thirds of them are in the form of layered structures where the in-plane bonds are strong (covalent), and the out-of-plane bonds are weak (van der Waals). This provides a means to mechanically or chemically thin (exfoliate) these materials down to a single atomic two-dimensional (2D) layer. While graphene, the 2D form of graphite, is metallic, the layered metal chalcogenides cover a wide range of electrical properties, from true metals $\left(\mathrm{NbS}_{2}\right)$ and superconductors $\left(\mathrm{TaS}_{2}\right)$ to semiconductors $\left(\mathrm{MoS}_{2}\right)$ with a wide range of bandgaps and offsets. Multiple techniques are currently being developed to synthesize large-area monolayers, including alloys, and lateral and vertical heterostructures. The wide range of properties and the ability to tune them on an atomic scale has led to numerous applications in electronics, optoelectronics, sensors, and energy. This article provides an introduction to TMDCs, serving as a background for the articles in this issue of MRS Bulletin.
\end{abstract}

\section{Introduction}

There are a wide variety of materials that exhibit layered structures. These materials (e.g., graphite, boron nitride, and molybdenum disulfide) lend themselves very well to being mechanically cleaved along the layers, which led to the utilization of these materials as dry lubricants. ${ }^{1}$ The last 10 years have shown us that these materials are also incredibly interesting for electronic and optoelectronic applications. ${ }^{2,3}$ By continuous cleaving of these layered materials, one can readily thin them down to a single layer, ${ }^{4,5}$ which results in the realization of new properties different from the bulk. These single layers, commonly known as two-dimensional (2D) materials, are receiving significant consideration due to their unique and tunable material properties: ${ }^{2}(1)$ They have no surface dangling bonds. (2) They are an ideal quantum well with wide-ranging direct and indirect energy bandgaps. (3) They can be superconducting or magnetic. (4) They can have enhanced spin-orbit coupling for applications in spin-based or valley-based electronics (i.e., use of the spin or momentum [valley] of an electron to encode the digital logic state). The unique properties of transition-metal dichalcogenides (TMDCs) are now being engineered to solve fundamental scientific and technological challenges.

\section{The bonds}

TMDCs are a subset of the layered materials family, where the intra-layer bonding is covalent and the inter-layer bonding consists of van der Waals (vdW) forces. This type of bond structure leads to highly anisotropic properties, where in-plane mechanical, thermal, and electrical properties significantly outperform the out-of-plane properties. ${ }^{6}$

Within a single plane, there are two types of crystal structures that dictate the electronic properties of the layers - trigonal prismatic $(2 \mathrm{H})$ and octahedral (1T). The trigonal prismatic structure is thermodynamically favorable for the majority of Mo and W-based TMDCs and leads to semiconducting behavior in the layers, while the octahedral phase is more stable for Hf and Zr-based TMDCs. ${ }^{7}$ While Hf and Zr-based TMDCs are semiconducting, many octahedrally bound TMDC layers (for example, $1 \mathrm{~T}-\mathrm{TaS}_{2}$ ) exhibit metallic or semi-metallic behavior, and each phase (trigonal or octahedral) can be transformed to the other via atomic gliding of the layers.

\section{A brief history}

Naturally occurring TMDCs (specifically $\mathrm{MoS}_{2}$ ) have been around for billions of years, ${ }^{8}$ but scientific publications on 
synthetic TMDCs did not begin to appear until the 1950 s. $^{9}$ The first reports of $\mathrm{MoS}_{2}$ synthesis were from the reduction of molybdenum trisulfide $\left(\mathrm{MoS}_{3}\right)^{9,10}$ and high temperature solution-based techniques. ${ }^{11}$ These initial works led to a wave of TMDC research from the 1960s to the 1980s, which primarily focused on the utilization of TMDCs for catalysis. During this time, there were hundreds of papers, books, and review articles written on the chemical synthesis (chemical vapor transport or deposition) and properties of TMDCs (too many to properly cite all). ${ }^{6,12-17}$ The first report of a single-layer TMDC was reported in $1986,{ }^{18}$ but this was followed by stagnation of the field until 2005 when Geim and Novoselov demonstrated mechanical exfoliation of graphite, boron nitride, and molybdenum disulfide (among other) layered materials to form monolayers, ${ }^{4}$ which exhibited very different properties than their bulk counterparts. Since then, the field of 2D materials has been a primary focus of many nanoscience and nanotechnology journals, with thousands of publications on the subject. ${ }^{19}$

\section{Synthesis of transition-metal dichalcogenides}

The earliest methods of synthesizing single layers of TMDCs include solution-based synthesis and chemical exfoliation of naturally occurring TMDCs; however, it was only in the 1970s when thin films of TMDCs were synthesized. The primary synthesis method was the conversion of a thin transition-metal $\mathrm{M}$ (Mo, W, etc.) or transition-metal oxide $\left(\mathrm{MoO}_{3}, \mathrm{WO}_{3}\right.$, etc. $)$ film into $\mathrm{MX}_{2}$ via exposure to a chalcogen vapor $\mathrm{X}(\mathrm{S}(\mathrm{g})$, $\mathrm{Se}(\mathrm{g}), \mathrm{H}_{2} \mathrm{~S}$, etc. $),{ }^{20-22}$ or reaction with solid chalcogen layers (Figure 1a). While this technique is quite successful at achieving continuous films of TMDs, it often results in nanocrystalline structures, though recent works indicate that this can be overcome to achieve high-quality multilayer films. ${ }^{23}$ On the other hand, many of the mechanically exfoliated flakes used today ${ }^{4,24-32}$ often come from bulk crystals that are synthesized via chemical vapor transport (CVT). ${ }^{33}$

CVT (Figure 1b) consists of mixing stoichiometric amounts of the transition metal with the chalcogen and a transport agent in a sealed vacuum tube. This system is then placed in a high-temperature furnace $\left(700-1000^{\circ} \mathrm{C}\right)$, where a temperature gradient across the sealed tube provides a driving force for transport of the elemental materials to the cool end, forming crystals of $\mathrm{MX}_{2} \cdot{ }^{34-36}$ While this technique provides a facile route to mechanical exfoliation of monolayer materials, it was not until the development of in situ powder vaporization (Figure 1c) that single crystal monolayer TMD materials were directly synthesized on insulating substrates. ${ }^{37}$ Most studies utilizing this method focus on vaporization of metal oxide $\left(\mathrm{MoO}_{3}, \mathrm{WO}_{3}\right.$, etc. $)$ and chalcogen $(\mathrm{S}, \mathrm{Se})$ powders in a horizontal tube furnace, where the chalcogen powder is placed upstream from the hot zone and just outside the tube furnace. ${ }^{38-42}$ Upon heating, the metal oxide and chalcogen powders are vaporized and transported downstream in the furnace, coating the substrate material and forming $\mathrm{MX}_{2}$ (Figure 1c). a

Thin-Film Conversion

$\mathrm{b}$
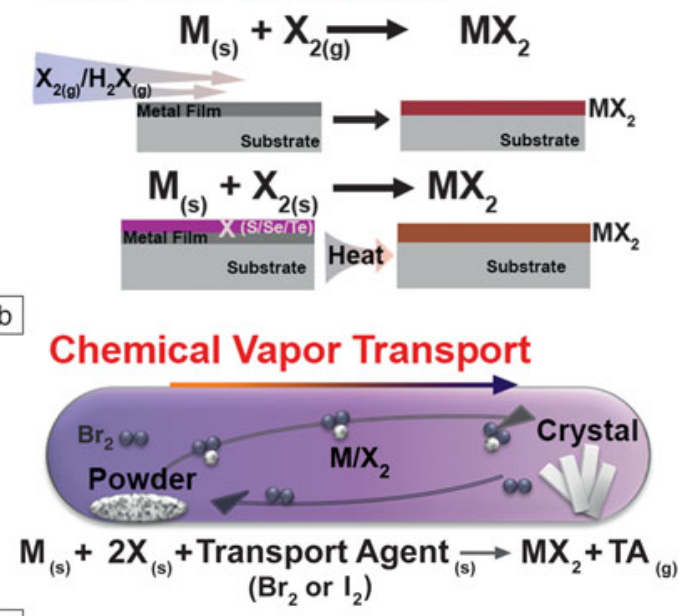

C

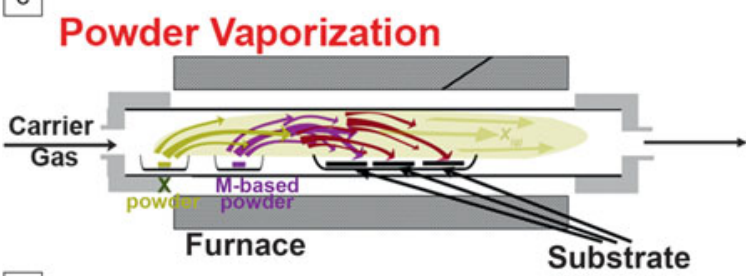

d

[MO] Chemical Vapor Deposition

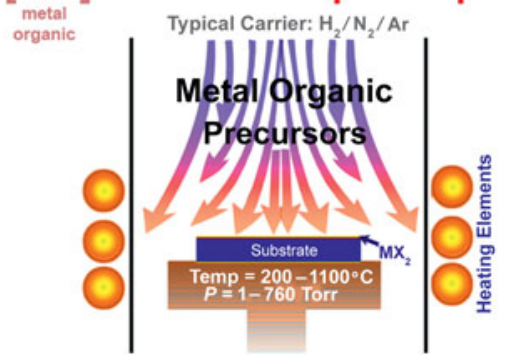

Figure 1. Various popular routes for the synthesis of $2 \mathrm{D}$ materials beyond graphene. (a) Thin-film conversion, where a solid film of metal or metal oxide is converted to an $\mathrm{MX}_{2}$ structure by exposing it to chalcogen vapors; (b) chemical vapor transport, where constituent powders of $\mathrm{M}$ and $\mathrm{X}$ are mixed with a transport agent (i.e., $\mathrm{Br}$ ) that assists the elements in vaporizing on the hot side of the growth vessel and recondensing on the cold side; (c) powder vaporization and transport, where powders of the constituent $\mathrm{M}$ and $\mathrm{X}$ are vaporized in a dynamic flow system to react and grow thin films of $2 \mathrm{D}$ materials; and (d) chemical vapor deposition, where chemical vapor precursors are used to synthesize the 2D materials. Adapted with permission from Reference 48. Note: M, transition metal; $\mathrm{X}$, chalcogen; TA, transport agent; $P$, pressure.

Finally, there are a variety of more sophisticated, industrially compatible, methods of synthesizing monolayer TMDCs as well. For example, molecular beam epitaxy has yielded a variety of monolayer TMDC films, with unprecedented ability for in situ characterization of the films during synthesis. Additionally, chemical vapor deposition (CVD), and more specifically, metal-organic CVD (Figure 1d) shows significant 
promise for a truly scalable synthesis technique for TMDCs. ${ }^{43}$ This technique provides a means to quantitatively control the metal and chalcogen precursor introduction, can be scaled to $>200 \mathrm{~mm}$ diameter wafers, and provides a way to strictly control the vapor-phase chemistry during synthesis - critical when tuning the properties of TMDCs for electronic and optical applications.

While the synthesis of these materials could lead to high impact science and advancements in technology, we still have a long way to go for controlling the stoichiometry of molybdenum-based (and other) TMDCs. Many reports discuss the synthesis of $\mathrm{MoS}_{2}$ and related TMDCs, however, $\mathrm{MoS}_{2}$ can exist over a wide range of sulfur atomic concentrations $(66 \rightarrow 69$ at. \%; see Figure 2 ) based on synthesis temperature. ${ }^{44-46}$ This translates to the possibility of growing the tetragonally bounded $(2 \mathrm{H})$ phase of $\mathrm{MoS}_{2}$ with stoichiometry up to $\mathrm{MoS}_{2.22}$ while still maintaining the octahedral structure. The story is quite similar for $\mathrm{MoSe}_{2}$ and $\mathrm{MoTe}_{2}$ (Figure 2), and this excess chalcogen ( $\mathrm{S}, \mathrm{Se}, \mathrm{Te}$ ) can significantly impact the physico-chemical properties of the layers and will undoubtedly affect one's ability to achieve controlled doping of such layers for electronic applications. Importantly, tungstenbased TMDCs do not have this same problem. Each W-based TMDC is stoiciometrically pure (a line compound), which is favorable for controlling the stoichiometry, but these materials are often more difficult to synthesize, requiring higher temperatures.

\section{The possibilities}

"What could we do with layered structures with just the right layers? What would the properties of materials be if we could really arrange the atoms the way we want them ..." ${ }^{47}$ These words from Richard Feynman started the scientific world down the path to discover 2D materials. Like their three-dimensional material counterparts (metals, silicon, $\mathrm{In}_{x} \mathrm{Ga}_{1-x} \mathrm{As}, \mathrm{Al}_{x} \mathrm{Ga}_{1-x} \mathrm{~N}$ etc.), monolayer 2D TMDCs have a wide range of properties from metallic and superconducting to semiconducting to magnetic with varying direct and indirect bandgaps..$^{2,3,48,49}$ Furthermore, alloying or straining of TMDCs can be used to vary the bandgap. Figure 3 shows the theoretical bandgap for $2 \mathrm{H}-\mathrm{MX}_{2(1-x)} \mathrm{X}_{2 x}^{\prime}$ as a function of concentration $\left(M=M o, W \text { and } X, X^{\prime}=S, S e, T e\right)^{50}$ and Figure 4 shows the theoretical bandgap of several 2H-TMDs as a function of lattice constant modified through application of $-5 \%$ to $+5 \%$ biaxial strain. ${ }^{51}$ While experimental verification of these theoretical bandgaps is continuing, the results suggest that a combination of alloying and strain can provide bandgaps in these TMDCs ranging from $0 \mathrm{eV}$ to as high as $\sim 2.0 \mathrm{eV}$.

Stacking of 2D TMDC monolayers also strongly impacts their properties. For example, monolayer $\mathrm{MoS}_{2}$ has a direct bandgap of $\sim 1.8 \mathrm{eV}$, whereas multilayers have a smaller indirect bandgap saturating at $\sim 1.2 \mathrm{eV}$ for bulk (greater than $\sim 5$ layers) $\operatorname{MoS}_{2} \cdot{ }^{52,53}$ The combination of materials used in a stack and associated interactions are also important. ${ }^{54}$ Although monolayer $\mathrm{MoS}_{2}$ and $\mathrm{WS}_{2}$ both have direct

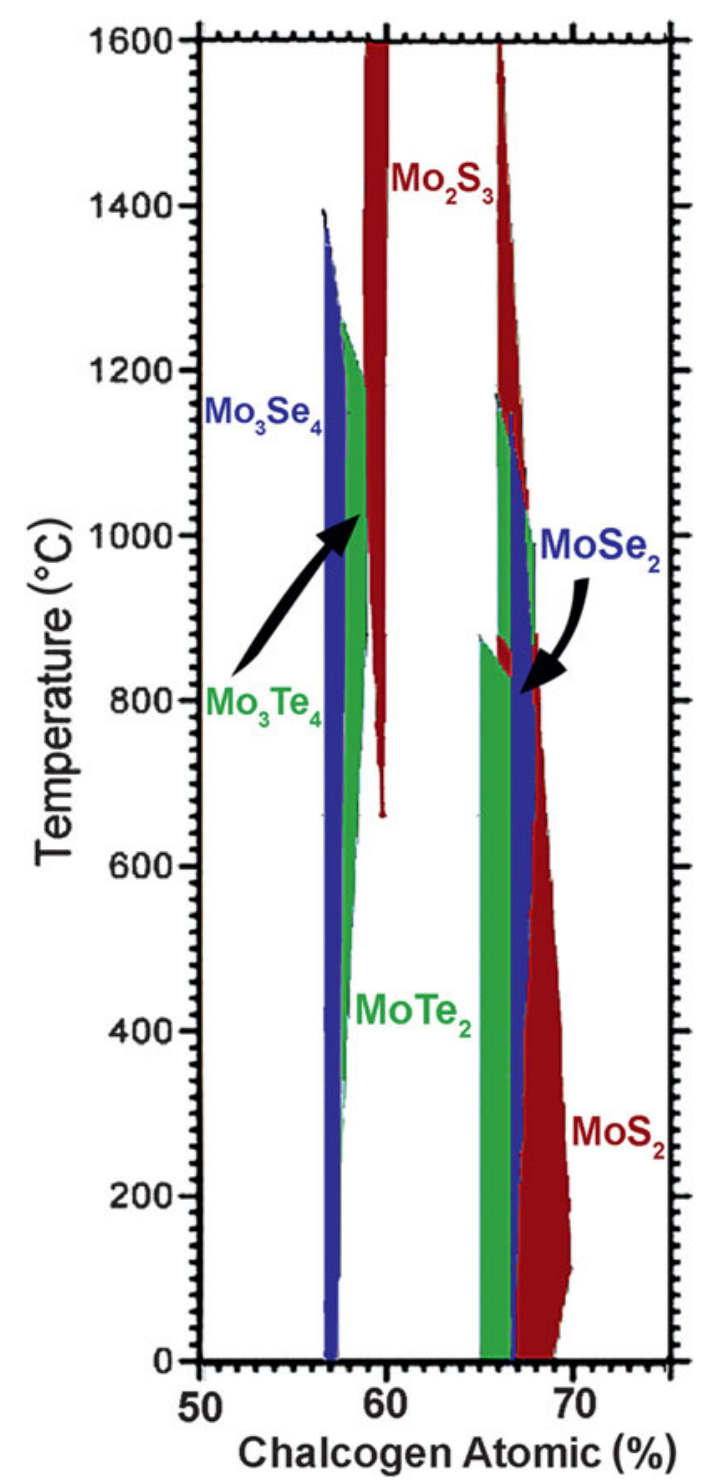

Figure 2. Partial phase diagram of molybdenum-based transition-metal dichalcogenide materials showing the wide miscibility ranges of each compound. This miscibility must be considered for the synthesis of two-dimensional transition-metal dichalcogenides. ${ }^{44-46}$

bandgaps, the bandgap of a $\mathrm{MoS}_{2} / \mathrm{WS}_{2}$ stack is indirect. On the other hand, a $\mathrm{MoS}_{2} / \mathrm{MoSe}_{2}$ stack is a direct gap with the valence band maximum associated with the $\mathrm{MoSe}_{2}$ and the conduction band minimum associated with the $\mathrm{MoS}_{2}$. Researchers have also developed a range of processes for doping 2D materials including assembling molecular monolayers onto the TMDC, which donate/accept electrons to/from the TMDC resulting in $n$-type/ $p$-type doping, ${ }^{55-59}$ doping based on ionic gating, ${ }^{60,61}$ and using conventional dopants that substitute for either the metal or chalcogen in the TMDC. ${ }^{62-64}$

While this "toolbox" of 2D TMDC properties and associated applications is interesting, conventional three-dimensional (3D) 


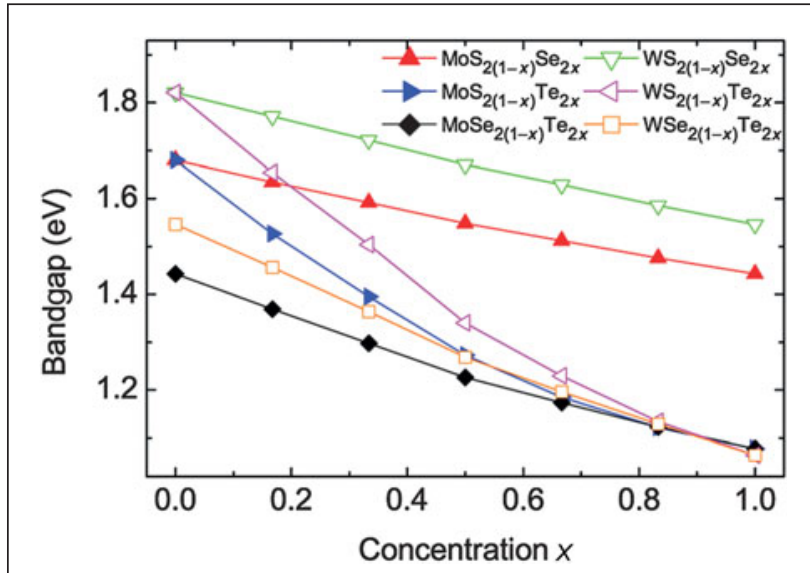

Figure 3. Theoretical bandgap of $\mathrm{MX}_{2(1-x)} \mathrm{X}_{2 x}^{\prime}$ monolayer alloys as a function of concentration. Alloying can be used to provide a continuous range of possible bandgaps and band offsets. Reprinted with permission from Reference 50. (C) 2013 AIP Publishing LLC. Note: $\mathrm{M}$, transition metal; $\mathrm{X}, \mathrm{X}^{\prime}$, chalcogen.

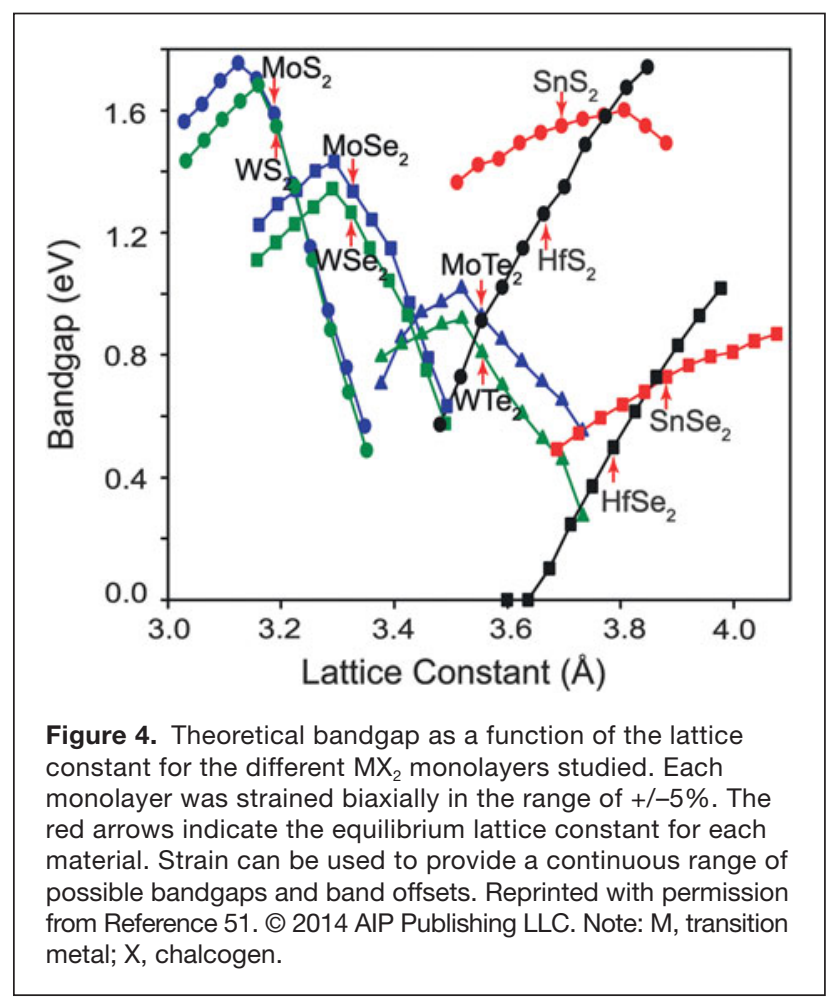

materials provide a similar parameter space. The properties of conventional 3D metals and semiconductors can also be modified using alloying and strain, and vertical heterostructures can be fabricated using epitaxy. So, the question is: What makes 2D TMDCs unique? The asymmetry of the in-plane covalent bonds compared to the out-of-plane vdW bonds for TMDCs results in properties not easily obtainable using 3D crystals. First of all, the unique bonding of TMDCs results in an absence of surface dangling bonds (surface states). For the case of very thin $3 \mathrm{D}$ semiconductors $(<\sim 10 \mathrm{~nm})$, control of these surface states through hydrogenation, oxidation, or lattice-matched epitaxy is crucial to ensure that surface states do not degrade the electronic/optical properties of the semiconductor. Also, the need for lattice matching during epitaxy of $3 \mathrm{D}$ materials limits the range of material combinations and achievable band offsets. This is critical, for example, in III-V or III-N resonant tunneling heterostructures where the low band offset between a quantum well and tunneling barrier results in thermionic emissions, which strongly contributes to the unwanted valley current. The vdW bond between 2D materials in vertical heterostructures allows for a wider range of materials that can be used. For example, hexagonal boron nitride (h-BN) with a large bandgap can be stacked with a monolayer TMD. Monolayer TMDCs are also an ideal quantum well in that they have only one longitudinal sub-band (one allowed energy level perpendicular to the material). Quantum wells of 3D semiconductors typically have multiple sub-bands associated with confinement effects, which strongly impact their electronic and optoelectronic properties.

The ability to define the layer property in a heterostructure based on the combination of a metal and chalcogen atom, which can be tuned from $>40$ binary combinations, ${ }^{49}$ and nearly countless alloy possibilities opens up an extremely broad range of electronic, optical, and other properties and applications. The presence of a bandgap (as compared to graphene), the ability to intimately control the band structure of the material, and the ability to inherently control the thickness of TMDCs down to a monolayer creates a wide variety of possible electronic, optoelectronic, and other applications. TMDCs can enhance the performance of conventional field-effect transistors for digital electronics since the monolayer thickness and lack of surface dangling bonds improves performance, electrostatics, and associated lateral scaling. ${ }^{2,24,27,29,31,65-67}$ Tuning the band structure of TMDCs permits development of novel low-power devices based on tunneling. ${ }^{68,69}$ As monolayer TMDCs have a direct bandgap, efficient optoelectronic devices such as light-emitting diodes and solar cells can be fabricated. $3,26,58,67,70$ The large surfaceto-volume ratio of these materials enables a variety of highly sensitive chemical and biological sensors. ${ }^{67,71-73}$ TMDCs also show large electroactivity, which is critical, for example, in catalysis. ${ }^{74-77}$

The relationship between electron energy and momentum in TMDCs is also different than that for many conventional semiconductors. Because of the lack of spatial symmetry and the presence of $d$-orbitals associated with the transitionmetal atoms, many TMDCs exhibit strong spin-orbit coupling, which is an interaction between the electron spin and orbital angular momentum. ${ }^{78-81}$ Spin-orbit coupling causes the energy of electrons with different spins to split and is useful in spintronic devices where the spin of the electron is used to carry information. The lack of symmetry also allows electrons or holes at the same energy but with different momentum (different band "valleys") to be independently populated. The use of 
spin or valley to carry information can potentially result in much lower-power electronic devices because the electron does not necessarily have to move through the material to carry information; information can instead be encoded using the electron's spin or valley.

We are now at the point where researchers can answer the questions originally posed by Feynman. We are now able to cleave and stack arbitrary atomic layers together to form complex material architectures, which could ultimately lead to the ability to "invent" a wealth of new properties based on the application at hand.

\section{In this issue}

The articles in this issue of MRS Bulletin provide a wide overview of the fundamental materials properties of TMDCs and their relationship to applications in electronics, optoelectronics, and energy. Shi et al. review the bulk and interfacial structure of TMDCs in detail. The relative merits of processes for synthesizing and modifying TMDCs are reviewed, including exfoliation, intercalation, vapor phase deposition, doping, and stacking. Kuc et al. review quantum calculations of the TMDC band structure to demonstrate the impact of electric field, strain, and stacking of layers on electronic properties. The application of TMDCs to transistors, circuits, and flexible devices is also presented. Chhowalla et al. describe the use of physical processes such as intercalation, doping, and heating to change the structural phase of TMDCs. The impact of these TMDCs phases on applications such as hydrogen evolution reactions, battery electrodes, and electronics is then discussed. Finally, Cao describes the properties of excitons in TMDCs including their binding energies, absorption, emission, and other dynamic properties. The engineering of these excitons to achieve interesting light-matter interactions and associated optoelectronic applications are described.

\section{Acknowledgments}

This work was partially supported by the Center for Low Energy Systems Technology, one of six centers supported by the STARnet phase of the Focus Center Research Program, a Semiconductor Research Corporation program sponsored by MARCO and DARPA. J.A.R. also acknowledges support from the National Science Foundation through the EFRI2DARE program (EFRI-1433307) and the Defense Threat Reduction Agency (HDTRA1-14-1-0037).

\section{References}

1. F.J. Clauss, Solid Lubricants and Self-Lubricating Solids (Elsevier, New York 1972).

2. G. Fiori, F. Bonaccorso, G. Iannaccone, T. Palacios, D. Neumaier, A. Seabaugh, S.K. Banerjee, L. Colombo, Nat. Nanotechnol. 9, 768 (2014).

3. Q.H. Wang, K. Kalantar-Zadeh, A. Kis, J.N. Coleman, M.S. Strano, Nat. Nanotechnol. 7, 699 (2012).

4. K.S. Novoselov, D. Jiang, F. Schedin, T.J. Booth, V.V. Khotkevich, S.V. Morozov, A.K. Geim, Proc. Natl. Acad. Sci. U.S.A. 102, 10451 (2005)

5. K.S. Novoselov, A.K. Geim, S.V. Morozov, D. Jiang, M.I. Katsnelson, I.V. Grigorieva, S.V. Dubonos, A.A. Firsov, Nature 438, 197 (2005).

6. F. Lévy, Ed., Crystallography and Crystal Chemistry of Materials with Layered Structures (Springer Netherlands, Dordrecht, 1976).
7. C. Gong. H. Zhang, W. Wang, L. Colombo, R.M. Wallace, K. Cho, Appl. Phys. Lett. 103, 053513 (2013).

8. J. Golden, M. McMillan, R.T. Downs, G. Hystad, I. Goldstein, H.J. Stein A. Zimmerman, D.A. Sverjensky, J.T. Armstrong, R.M. Hazen, Earth Planet. Sci. Lett. 366, 1 (2013).

9. F.T. Eggertsen, R.M. Roberts, J. Phys. Chem. 63, 1981 (1959).

10. H. Yagoda, H.A. Fales, J. Am. Chem. Soc. 58, 1494 (1936).

11. L.A. Arutyuny, E.K. Khurshud, Geochem. Int. USSR 3, 479 (1966).

12. J.A. Wilson, A.D. Yoffe, Adv. Phys. 18, 193 (1969).

13. T.J. Wieting, M. Schlüter, Eds., Electrons and Phonons in Layered Crystal Structures (Springer Netherlands, Dordrecht, 1979).

14. K. Motizuki, Ed., Structural Phase Transitions in Layered Transition Metal Compounds (Springer Netherlands, Dordrecht, 1986).

15. R.M.A. Lieth, Ed., Preparation and Crystal Growth of Materials with Layered Structures (Springer Netherlands, Dordrecht, 1977).

16. P.A. Lee, Ed., Optical and Electrical Properties (Springer Netherlands, Dordrecht, 1976)

17. V. Grasso, Ed., Electronic Structure and Electronic Transitions in Layered Materials (Springer Netherlands, Dordrecht, 1986).

18. P. Joensen, R.F. Frindt, S.R. Morrison, Mater. Res. Bull. 21, 457 (1986).

19. Nat. Mater. 10, 1 (2011).

20. A. Khelii, H. Essaidi, J. Bernede, J. Pouzet, M. Mebarki, Ann. Chimie. Sci. Materiaux 21, 295 (1996).

21. A. Khelil, H. Essaidi, J.C. Bernede, A. Bouacheria, J. Pouzet, J. Phys. Condens. Matter 6, 8527 (1994).

22. H. Hadouda, J. Pouzet, J.C. Bernede, A. Barreau, Mater. Chem. Phys. 42, 291 (1995)

23. A. Tarasov, P.M. Campbell, M.-Y. Tsai, Z.R. Hesabi, J. Feirer, S. Graham, W.J. Ready, E.M. Vogel, Adv. Funct. Mater. 24, 6389 (2014).

24. G.A. Salvatore, N. Münzenrieder, C. Barraud, L. Petti, C. Zysset, L. Büthe, K. Ensslin, G. Tröster, ACS Nano 7, 8809 (2013).

25. X. Xie, D. Sarkar, W. Liu, J. Kang, O. Marinov, M. Jamal Deen, K. Banerjee, ACS Nano 8, 5633 (2014).

26. H. Nan, Z. Wang, W. Wang, Z. Liang, Y. Lu, Q. Chen, D. He, P. Tan, F. Miao, X. Wang, J. Wang, Z. Ni, ACS Nano 8, 5738 (2014).

27. J. Yoon, W. Park, G.-Y. Bae, Y. Kim, H.S. Jang, Y. Hyun, S.K. Lim, Y.H. Kahng, W.-K. Hong, B.H. Lee, H.C. Ko, Small 9, 3295 (2013)

28. A. Splendiani, L. Sun, Y. Zhang, T. Li, J. Kim, C.-Y. Chim, G. Galli, F. Wang, Nano Lett. 10, 1271 (2010).

29. S. Das, H.-Y. Chen, A.V. Penumatcha, J. Appenzeller, Nano Lett. 13, 100 (2013) 30. H. Fang, S. Chuang, T.C. Chang, K. Takei, T. Takahashi, A. Javey, Nano Lett. 12, 3788 (2012)

31. W. Liu, J. Kang, D. Sarkar, Y. Khatami, D. Jena, K. Banerjee, Nano Lett. 13, 1983 (2013)

32. H. Terrones, E. Del Corro, S. Feng, J.M. Poumirol, D. Rhodes, D. Smirnov, N.R. Pradhan, Z. Lin, M.A.T. Nguyen, A.L. Elías, T.E. Mallouk, L. Balicas, M.A. Pimenta, M. Terrones, Sci. Rep. 4, 4215 (2014).

33. J. Mercier, J. Cryst. Growth 56, 235 (1982).

34. Y. Liu, R. Ang, W.J. Lu, W.H. Song, L.J. Li, Y.P. Sun, Appl. Phys. Lett. 102 $192602(2013)$

35. R. Ang, Y. Miyata, E. leki, K. Nakayama, T. Sato, Y. Liu, W.J. Lu, Y.P. Sun, T. Takahashi, Phys. Rev. B: Condens. Matter 88, 115145 (2013).

36. A.A. Al-Hilli, B.L. Evans, J. Cryst. Growth 15, 93 (1972)

37. Y.-C. Lin, W. Zhang, J.-K. Huang, K.-K. Liu, Y.-H. Lee, C.-T. Liang, C.-W. Chu, L.-J. Li, Nanoscale 4, 6637 (2012).

38. Y. Zhan, Z. Liu, S. Najmaei, P.M. Ajayan, J. Lou, Small 8, 966 (2012).

39. Y.-H. Lee, X.-Q. Zhang, W. Zhang, M.-T. Chang, C.-T. Lin, K.-D. Chang, Y.-C. Yu, J.T.-W. Wang, C.-S. Chang, L.-J. Li, T.-W. Lin, Adv. Mater. 24, 2320 (2012). 40. Y. Yu, C. Li, Y. Liu, L. Su, Y. Zhang, L. Cao, Sci. Rep. 3, 1866 (2013).

41. A.L. Elías, N. Perea-López, A. Castro-Beltrán, A. Berkdemir, R. Lv, S. Feng A.D. Long, T. Hayashi, Y.A. Kim, M. Endo, H.R. Gutiérrez, N.R. Pradhan, L. Balicas, T.E. Mallouk, F. López-Urías, H. Terrones, M. Terrones, ACS Nano 7, 5235 (2013).

42. H.R. Gutiérrez, N. Perea-López, A.L. Elías, A. Berkdemir, B. Wang, R. Lv, F. López-Urías, V.H. Crespi, H. Terrones, M. Terrones, Nano Lett. 13, 3447 (2013).

43. S.M. Eichfeld, L. Hossain, Y.-C. Lin, A.F. Piasecki, B. Kupp, A.G. Birdwell, R.A. Burke, N. Lu, X. Peng, J. Li, A. Azcatl, S. McDonnell, R.M. Wallace, M.J. Kim T.S. Mayer, J.M. Redwing, J.A. Robinson, ACS Nano 9, 2080 (2015).

44. L. Brewer, R.H. Lamoreaux, in Binary Alloy Phase Diagrams, 2nd ed. T.B. Massalski, Ed. (ASM International, Materials Park, OH, 1990), vol. 3, pp. $2660-2661$

45. L. Brewer, R.H. Lamoreaux, in Binary Alloy Phase Diagrams, 2nd ed., T.B. Massalski, Ed. (ASM International, Materials Park, OH, 1990), pp. 2664-2665. 46. L. Brewer, R.H. Lamoreaux, in Binary Alloy Phase Diagrams, 2nd ed., T.B. Massalski, Ed. (ASM International, Materials Park, OH, 1990), pp. 2675-2676. 47. R. Feynman, American Physical Society Annual Meeting (American Physical Society, California Institute of Technology, 1959). 
48. S. Das, J.A. Robinson, M. Dubey, H. Terrones, M. Terrones, Annu. Rev. Mater. Res. 45 (forthcoming), available at http://www.annualreviews.org/doi/ abs/10.1146/annurev-matsci-070214-021034.

49. M. Chhowalla, H.S. Shin, G. Eda, L.-J. Li, K.P. Loh, H. Zhang, Nat. Chem. 5, $263(2013)$

50. J. Kang, S. Tongay, J. Li, J. Wu, J. Appl. Phys. 113, 143703 (2013).

51. D.M. Guzman, A. Strachan, J. Appl. Phys. 115, 243701 (2014).

52. J. Kang, S. Tongay, J. Zhou, J. Li, J. Wu, Appl. Phys. Lett. 102, 012111 (2013).

53. J.E. Padilha, H. Peelaers, A. Janotti, C.G. Van de Walle, Phys. Rev. B: Condens. Matter 90, 1 (2014)

54. H.P. Komsa, A.V. Krasheninnikov, Phys. Rev. B: Condens. Matter 88, 1 (2013).

55. D. Kiriya, M. Tosun, P. Zhao, J.S. Kang, A. Javey, J. Am. Chem. Soc. 136, 7853 (2014).

56. P. Zhao, D. Kiriya, A. Azcatl, C. Zhang, M. Tosun, Y.-S. Liu, M. Hettick, J.S. Kang, S. McDonnell, K.C. Santosh, J. Guo, K. Cho, R.M. Wallace, A. Javey, ACS Nano 8, $10808(2014)$

57. L. Yang, K. Majumdar, H. Liu, Y. Du, H. Wu, M. Hatzistergos, P.Y. Hung, R. Tieckelmann, W. Tsai, C. Hobbs, P.D. Ye, Nano Lett. 14, 6275 (2014).

58. B.W.H. Baugher, H.O.H. Churchill, Y. Yang, P. Jarillo-Herrero, Nat. Nanotechnol. 9,262 (2014)

59. A. Tarasov, S. Zhang, M.-Y. Tsai, P.M. Campbell, S. Graham, S. Barlow, S.R. Marder, E.M. Vogel, Adv. Mater. 27, 1175 (2015)

60. N. Ubrig, S. Jo, H. Berger, A.F. Morpurgo, A.B. Kuzmenko, Appl. Phys. Lett. 104, $171112(2014)$

61. S. Fathipour, H. Xu, E. Kinder, S. Fullerton-Shirey, A. Seabaugh, IEEE Device Res. Conf. (DRC) Proc. (IEEE, New York, 2014), pp. 125-126.

62. J. Suh, T.-E. Park, D.-Y. Lin, D. Fu, J. Park, H.J. Jung, Y. Chen, C. Ko, C. Jang, Y. Sun, R. Sinclair, J. Chang, S. Tongay, J. Wu, Nano Lett. 14, 6976 (2014). 63. A. Carvalho, A.H.C. Neto, Phys. Rev. B: Condens. Matter 89, 081406 (2014)

64. H.-P. Komsa, N. Berseneva, A.V. Krasheninnikov, R.M. Nieminen, Phys. Rev. X 4, 031044 (2014)
65. T. Roy, M. Tosun, J.S. Kang, A.B. Sachid, S.B. Desai, M. Hettick, C.C. Hu, A. Javey, ACS Nano 8, 6259 (2014).

66. K. Majumdar, C. Hobbs, P.D. Kirsch, IEEE Electron Device Lett. 35, 402 (2014)

67. D. Jariwala, V.K. Sangwan, L.J. Lauhon, T.J. Marks, M.C. Hersam, ACS Nano 8, $1102(2014)$

68. K. Lam, X. Cao, J. Guo, S. Member, IEEE Electron Device Lett. 34, 1331 (2013)

69. T. Yamaguchi, R. Moriya, Y. Inoue, S. Morikawa, S. Masubuchi, K. Watanabe, T. Taniguchi, T. Machida, Appl. Phys. Lett. 105, 223109 (2014).

70. M.-L. Tsai, S.-H. Su, J.-K. Chang, D.-S. Tsai, C.-H. Chen, C.-I. Wu, L.-J. Li, L.-J. Chen, J.-H. He, ACS Nano. 8, 8317 (2014).

71. M. Donarelli, S. Prezioso, F. Perrozzi, F. Bisti, M. Nardone, L. Giancaterini, C. Cantalini, L. Ottaviano, Sens. Actuators B Chem. 207, 602 (2015)

72. D.J. Late, T. Doneux, M. Bougouma, Appl. Phys. Lett. 105, 233103 (2014).

73. D. Sarkar, W. Liu, X. Xie, A.C. Anselmo, S. Mitragotri, K. Banerjee, ACS Nano 8, 3992 (2014)

74. A.Y.S. Eng, A. Ambrosi, Z. Sofer, P. Simek, M. Pumera, ACS Nano 8, 12185 (2014)

75. L. Guardia, J.I. Paredes, J.M. Munuera, S. Villar-Rodil, M. Ayan-Varela, A. Martinez-Alonso, J.M.D. Tascon, ACS Appl. Mater. Interfaces 6, 21702 (2014).

76. X. Geng, W. Wu, N. Li, W. Sun, J. Armstrong, A. Al-hilo, M. Brozak, J. Cui, T. Chen, Adv. Funct. Mater. 24, 6123 (2014).

77. D. Voiry, M. Salehi, R. Silva, T. Fujita, M. Chen, T. Asefa, V.B. Shenoy, G. Eda, M. Chhowalla, Nano Lett. 13, 6222 (2013).

78. N. Zibouche, P. Philipsen, A. Kuc, T. Heine, Phys. Rev. B: Condens. Matter 90, 125440 (2014)

79. H. Yuan, X. Wang, B. Lian, H. Zhang, X. Fang, B. Shen, G. Xu, Y. Xu, S.-C. Zhang, H.Y. Hwang, Y. Cui, Nat. Nanotechnol. 9, 851 (2014).

80. J.M. Riley, F. Mazzola, M. Dendzik, M. Michiardi, T. Takayama, L. Bawden, C. Granerod, M. Leandersson, T. Balasubramanian, M. Hoesch, T.K. Kim, H. Takagi, W. Meevasana, P. Hofmann, M.S. Bahramy, J.W. Wells, P.D.C. King, Nat. Phys. 10, 835 (2014)

81. F. Guinea, M.I. Katsnelson, T.0. Wehling, Ann. Phys. 526, A81 (2014).

\section{NEW Advances in X-ray Diffraction}

\section{OD, 1D and 2D}

All from a single Hybrid Pixel Array Detector (HPAD)

- Ultra-high dynamic range and high sensitivity

- Seamless switching from 2D-TDI mode to 2D snapshot mode to 1D-TDI mode to OD mode with a single detector

- XRF suppression by high and low energy discrimination

- High spatial resolution, direct-detection pixel array detector

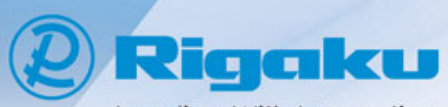

Leading With Innovation

Rigaku Corporation and its Global Subsidiaries www.Rigaku.com | info@Rigaku.com

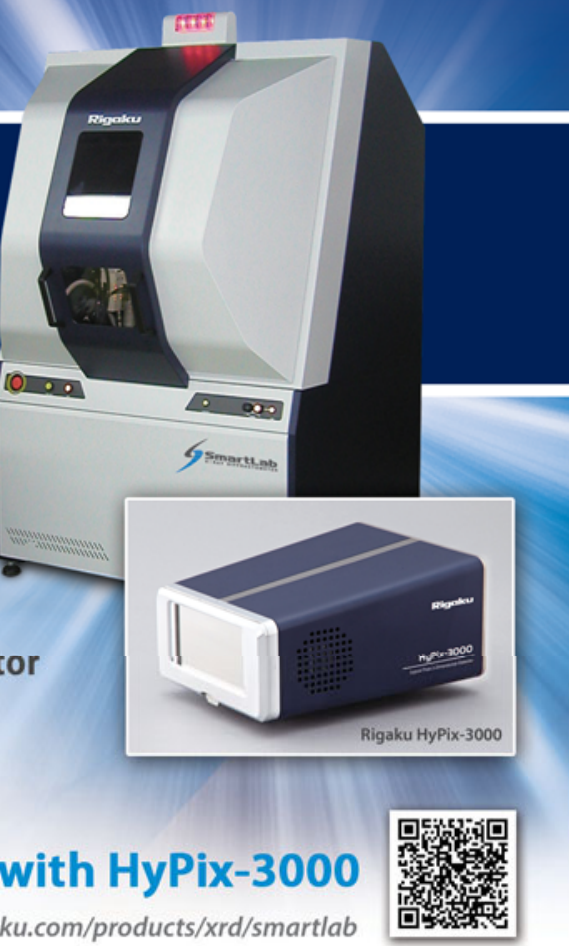

